\title{
Parasitism by the "Slow" Bacterium Helicobacter pylori Leads to Altered Gastric Homeostasis and Neoplasia
}

\author{
Martin J. Blaser* and Julie Parsonnet* \\ *Departments of Medicine and Microbiology and Immunology, Vanderbilt University School of Medicine and Veterans Affairs Medical \\ Center, Nashville, Tennessee 37232; and ${ }^{\ddagger}$ Departments of Medicine and Health Research and Policy, Stanford University School of \\ Medicine, Stanford, California 94305
}

Nature uses as little of anything as possible.

\section{Johannes Kepler}

Helicobacter pylori are gram negative bacteria that live in the human stomach. They may be considered "slow" bacterial pathogens because of their ability to persist in this seemingly hostile environment for decades if not for life, because they induce an inflammatory response, and because this interaction may lead to a variety of clinical consequences, among them peptic ulceration and gastric neoplasia. It is important to understand the biology of $H$. pylori infections, not only because it is involved in the pathogenesis of two of the most important diseases of the upper gastrointestinal tract, peptic ulcer disease and adenocarcinoma of the stomach, but because it also serves as a model of the effects of chronic mucosal inflammation on endocrine homeostasis and on oncogenesis.

However, $H$. pylori infection also allows us to view the consequences of persistence from the context of the successful parasite as well. We know that, if the range of a microbe is limited to a single species or a closely related group of species, there is a strong negative selective pressure against those that are overwhelmingly virulent; the central concern of the single reservoir microbe is the ability to be transmitted from host to host (1). The organism must strike a balance with regard to its virulence, such that a microbial density is produced that maximizes transmission in the normal host. All such balances involve costs (to the microbe); one cost is that, when a host with suboptimal defenses is encountered, disease may ensue that limits the ability of the microbe to find a new host.

$H$. pylori appears to have successfully met these challenges. At least a third of the world's human population is infected (2), usually beginning early in life, and the major disease consequences appear to be expressed years or decades after infection is acquired (3), diminishing negative selection against infection. This success probably was not won overnight, but rather reflects a long evolutionary history of coexistence of humans and their gastric bacterial parasites. Persistent infections, especially those in which an immune response indicates that the host is aware of the microbe's presence, must have a complex

Address correspondence to Martin J. Blaser, Division of Infectious Diseases, A-3310 Medical Center North, Vanderbilt University School of Medicine, Nashville, TN 37232.

Received for publication 11 March 1994 and in revised form 23 March 1994.

The Journal of Clinical Investigation, Inc.

Volume 94 , July $1994,4-8$ pathogenesis. Nevertheless, principles of natural selection and parsimony help in understanding their characteristics.

\section{Natural history of $\boldsymbol{H}$. pylori infection}

A decade after its rediscovery (4), we now have a reasonable understanding of the natural history of $\boldsymbol{H}$. pylori infection (Fig. 1). After ingestion, there is a period of intense bacterial proliferation and gastric inflammation. Symptoms referable to the upper gastrointestinal tract may be transiently present, and the immune response takes at least weeks to develop. Concomitant with the intense gastritis is hypochlorhydria, which may last for months (5). If animal models and inferences from human childhood infection are relevant (6), fecal shedding of $H$. pylori is maximal during this period, facilitating transmission to new hosts. Ultimately, the inflammatory response is reduced to a low-level stable state (termed chronic diffuse superficial gastritis), the host mounts a humoral immune response that is ineffective in eliminating $H$. pylori, normal gastric $\mathrm{pH}$ is restored, and the infected person becomes asymptomatic. This outcome persists for years or decades and appears to predominate in the population. A subset of infected persons develop peptic ulceration, and a very small proportion may develop gastric lymphoma. However, in many hosts, there is a gradual progression of the inflammatory process to affect gastric glandular structures leading to atrophic gastritis. This progression requires three to four decades on average (7) and is significant because atrophic gastritis and its concomitant intestinal metaplasia may be considered as premalignant lesions for the development of gastric adenocarcinoma (8). The development of atrophic gastritis also may lead to a progressive decline in $H$. pylori population numbers and its ultimate loss (9). The hypochlorhydria associated with this stage may both facilitate gastric colonization by other enteric organisms and provide a final opportunity for fecal transmission. Thus, it is interesting to speculate that, like Mycobacterium tuberculosis and varicella-zoster virus, $H$. pylori may have opportunities for transmission to new hosts both early and late in life.

\section{Persistence of $H$. pylori}

The majority of $H$. pylori organisms are free-living in the mucus layer of the stomach overlaying the mucosal epithelium, a small proportion appear to adhere to the epithelial cells, and few, if any, actually invade tissue. Thus, $H$. pylori are chiefly, if not exclusively, lumenal organisms and persist there despite the low $\mathrm{pH}$ and digestive enzymes present. Furthermore, $\boldsymbol{H}$. pylori must contend with peristalsis and the continuous shedding of both the mucus layer and the epithelial cells. Despite these adverse circumstances, $H$. pylori populations are maintained at high concentrations ( $\sim 10^{8} \mathrm{CFU} / \mathrm{g}$ ) for decades. As such, $H$. 


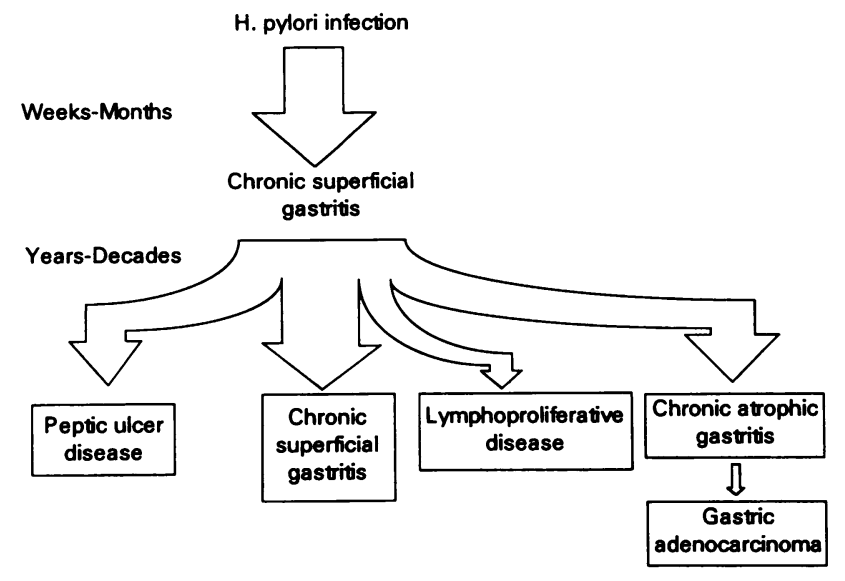

Figure 1 . Natural history of $H$. pylori infection. Within months after $H$. pylori acquisition, chronic superficial gastritis develops. In the absence of antimicrobial treatment, this process persists in most hosts for life. A minority of hosts may develop clinically relevant outcomes such as peptic ulceration, lymphoproliferative diseases, or severe chronic atrophic gastritis leading to adenocarcinoma of the distal stomach.

pylori cells must be actively replicating to sustain its numbers, and this lifestyle should be contrasted with several other persistent pathogens, such as $M$. tuberculosis or varicella-zoster virus, in which the preponderance of their tenure in the human host is as dormant foci. The survival of $H$. pylori is aided by at least two important factors. First, there is essentially no microbial competition in the stomach. Other than $H$. pylori and certain closely related organisms (e.g., Helicobacter heilmanii), the normal stomach has few other resident microbes. Second, although there is both humoral and cellular recognition of $H$. pylori $(10,11)$, it is apparently ineffective. Why $H$. pylori cannot be cleared by the immune system is presently unknown but evasion of the host immune response is the sine qua non of persistent parasitism. In a teleologic sense, it may be that the human host relies on gastric acidity and peristalsis to clear the stomach of microbes and thus has not evolved an adequate immune effector system for that location. Hence, it may be the ability to survive in an otherwise hostile milieu that is both necessary and sufficient for persistence.

$H$. pylori have evolved a number of mechanisms that facilitate persistence. Microaerophilic metabolism enables survival in the semipermeable mucus gel, and spiral shape, motility, and the ability to adhere to epithelial cells favor resistance to peristalsis (12). H. pylori possess large amounts of urease, enabling hydrolysis of urea to ammonia and carbon dioxide (13). Urease may serve several functions for $H$. pylori but most importantly, in the presence of urea, wild-type $\left(\mathrm{U}^{+}\right) H$. pylori withstand low $\mathrm{pH}$ whereas $\mathrm{U}^{-}$cells cannot (14). Thus, paradoxically, an acidic milieu is favorable for $H$. pylori since it is adapted for survival, whereas the competing microbes of the gastrointestinal tract are not. Further, it may be argued that mechanisms for maintenance of high intragastric acidity are adaptive for $H$. pylori so long as lethal (to the host) ulcerations are not produced. This hypothesis is consistent with the observation that $H$. pylori infection induces hypergastrinemia (15), which is a stimulant of parietal cell hydrochloric acid production. In addition, $\boldsymbol{H}$. pylori produce both superoxide dismutase and catalase which may prevent damage by phagocyte-released free oxygen radicals.

\section{H. pylori-induced inflammation}

A critical question is how $H$. pylori, which are essentially lumenal (off-shore) organisms, induce inflammation within the gastric mucosa. Analysis is complicated by the observations that gastric inflammation is uneven in affected persons, with marked variation in the extent of local involvement within an individual stomach, and no direct correlation between the local density of bacterial colonization and the extent or nature of the inflammatory response. However, much has been learned. We now know that $H$. pylori interact with gastric epithelial cells. Whether the effects on cells result from release of products from free-living organisms or as a result of adherence or possibly localized invasion is not known. Epithelial cells express class II molecules which may aid in presentation of $H$. pylori antigens and express cytokines such as IL-8 that might stimulate an inflammatory response.

Although $H$. pylori cells are not present in the lamina propria, $H$. pylori antigens including urease have been detected (16). H. pylori may release extracellular products that recruit inflammatory cells such as monocyte/macrophages and neutrophils (16), and their activation leads to release of mediators including interleukins, TNF- $\alpha$, and superoxide (17). Biopsies from $H$. pylori-infected persons show increased numbers of phagocytic cells (neutrophils and macrophages), plasma cells, and lymphocytes and increased expression of cytokines such as TNF- $\alpha$, IL-6, IL-1 $\beta, \mathrm{IL}-8$, and IL-10 (18). An inflammatory response persists for the duration of the infection; that antimicrobial treatment that eradicates $H$. pylori relieves the inflammation indicates the crucial role of the bacterium in its causation (19).

The persistence of an inflammatory response to a noninvasive organism seems maladaptive for the host since inflammation per se is destructive; evolution may have selected for hosts who downregulate the inflammatory response to $H$. pylori, just as hypothesized for other persistent pathogens (20). Evidence is accumulating that the cellular immune response to $H$. pylori is suppressed or downregulated in relation to the humoral response $(11,21)$, consistent with a TH2 rather than a TH1 immune response. Inflammation also might not seem beneficial for $H$. pylori, especially since the early stage of intense inflammation results in hypochlorhydria reducing an important selective advantage of $H$. pylori. Similarly, ongoing active inflammation enhances the risk of progression to atrophic gastritis with ultimate loss of a niche for $H$. pylori $(9,12)$. If inflammation is not beneficial for $H$. pylori, natural selection might favor organisms that induce little inflammation; that the lipopolysaccharide of $H$. pylori, like that of Bacteroides fragilis (another persistent mucosal organism), has low biological activity is consistent with this hypothesis.

Since inflammation is not obviously advantageous to either the host or the pathogen, why is it present? One hypothesis that has been raised is that $H$. pylori uses the inflammatory response as a means of obtaining a constant and reliable source of nutrients $(3,12)$. In this model (Fig. 2$), H$. pylori releases proinflammatory effectors that induce tissue inflammation, which causes release of nutrients that sustain the $H$. pylori population. This is a positive feedback loop, but both host downregulation of inflammation and bacterial signal transduction to downregulate effector release would operate to maintain the system over time (12). Mathematical analysis indicates that such a model can lead to steady states persisting for decades (Kirschner, 


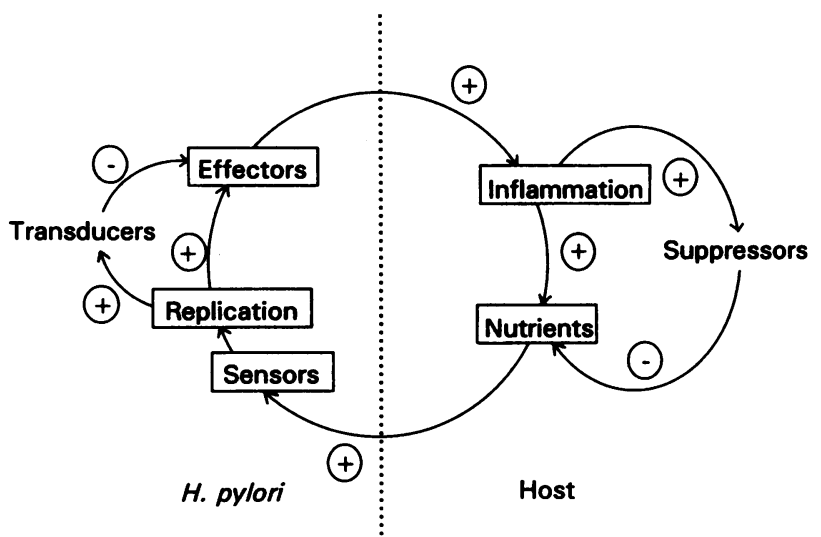

Figure 2. Model of $H$. pylori persistence based on proinflammatory activity. In this model, induction of inflammation by $H$. pylori is adaptive in the short run, in that inflammation will lead to release of nutrients for this lumenal organism. However, brisk inflammation leading to gastric atrophy is unfavorable for both host and microbe. It is postulated that chronic infection leads to downregulation of host inflammatory responses and that $\boldsymbol{H}$. pylori, when sated, diminishes release of proinflammatory effector molecules. These simultaneous downregulatory mechanisms act to minimize tissue injury and to assure perpetuation of the infection.

D. E., and M. J. Blaser, unpublished data). In total, the system proposed is highly regulated, in which modulation of the inflammatory response serves both host and parasite.

\section{Reasons for variability in outcome of $\boldsymbol{H}$. pylori infections}

Three clinical diseases have been linked to $H$. pylori infection: peptic ulcer disease (including both duodenal and gastric ulcers ), gastric adenocarcinoma, and gastric lymphoma (22). The majority of infected subjects, therefore, have no adverse consequences of their infection and remain asymptomatic throughout life (2). To date, no benefits of $\boldsymbol{H}$. pylori infection have been identified; however, because of its high prevalence, attention must be focused not only on deleterious consequences to the host but on possible advantages as well. Differences in clinical outcome can be explained, as follows.

Differences among $H$. pylori strains. $H$. pylori strains are extremely diverse at the nucleotide level, even for highly conserved genes. The reasons for genomic diversity are not known, but lack of other organisms in the stomach with which to exchange genetic information may place a premium on self-generation of variation. We now know of three $H$. pylori characteristics that are not conserved, that often occur in the same strains, that are each associated with increased inflammation, and that are associated independently with peptic ulceration (23-28). The first is a gene called cagA, which encodes a cryptic protein of 120-140 kD; the range in molecular mass is related to the number of intragenic repeats (23). Only $\sim 60 \%$ of $\mathrm{H}$. pylori strains possess cagA, but of the subset associated with idiopathic (non-drug-induced, non-Zollinger-Ellison syndrome) peptic ulceration, nearly all are positive $(24,25)$. A second property present in $50-60 \%$ of strains is expression of an 87$\mathbf{k D}$ cytotoxin that induces vacuolation in eukaryotic cells (26). In contrast to $\operatorname{cag} A$, the gene for the protoxin, called vacA, is present in all strains, but tox ${ }^{-}$strains have significant sequence divergence from tox ${ }^{+}$strains (27). A third property is the ability of strains to activate neutrophils in the absence of opsonins; the

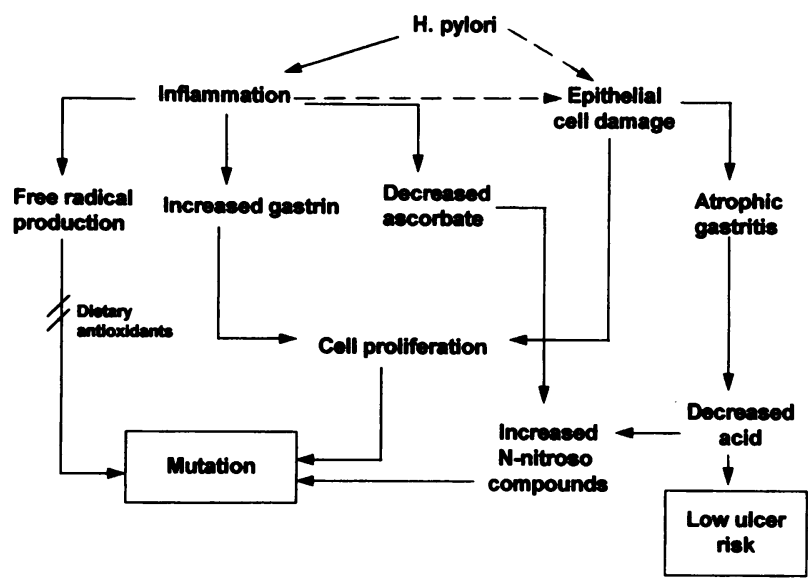

Figure 3. Model for the interaction of environmental factors and $\boldsymbol{H}$. pylori infection in gastric carcinogenesis. $H$. pylori, by causing inflammation and cell proliferation, enhances the likelihood of mutation in gastric epithelial cells.

basis for this property has not been established (28). Considering the diversity among $H$. pylori strains, it is likely that further differences will be found, and it is possible that subspecies will be formally identified.

Differences in host responses to $H$. pylori infection. It is axiomatic that, among outbred human populations, host responses to infectious agents are varied. Certain microbes causing high mortality, such as Plasmodium falciparum, exert a profound selective effect on human genes, but based on expression of $H$. pylori-related mortality largely after reproductive age a major selective effect is unlikely. Investigations involving monozygotic and dizygotic twins indicated a genetic component for peptic ulcer disease, and recent studies considering $H$. pylori infection are consistent (29). For example, familial clustering of gastric cancer and peptic ulceration could relate to the ability to downregulate inflammation. Persons with blood group $\mathrm{O}$ have an increased risk of peptic ulceration; recent work suggests that enhanced ability of $H$. pylori to adhere to cells bearing blood group $\mathrm{O}$ carbohydrate antigens may be related to that observation (30).

Environmental cofactors. Environmental determinants of gastric diseases had been recognized well before the first isolation of $H$. pylori. Dietary salts and nitrates (nitrates can be converted in vivo to mutagenic $N$-nitroso compounds) have been consistently, although not universally, implicated as gastric cancer risk factors $(31,32)$. In contrast, subjects who consume high quantities of fresh fruits and vegetables are protected against this malignancy. While consumption of several antioxidant vitamins, beta-carotene, ascorbic acid, and alpha-tocopherol, has been associated with disease protection, none yet have been proven in a clinical trial to prevent malignancy (32).

Development of gastric cancer probably depends on an interaction between these environmental cofactors and infection (Fig. 3). The chief role of $H$. pylori in carcinogenesis may be stimulating cell growth. Gastric epithelial cells proliferate more rapidly in patients with $H$. pylori infection than in uninfected hosts, either as a direct consequence of cell damage (33) or in response to hypergastrinemia (gastrin is trophic to the gastric epithelium [34]). Enhanced cell proliferation increases the likelihood of DNA damage in response to environmental mutagens 
such as $\mathrm{N}$-nitrosamines or inflammation-related free radicals $(35,36)$. Such damage could be minimized by antinitrosating agents (i.e., ascorbic acid) or antioxidants (i.e., beta-carotene or alpha-tocopherol). A role of cofactors similarly has been proposed for other malignancies occurring after chronic infections. For example, papilloma viruses cause cervical cancer in conjunction with chemical cofactors (i.e., cigarette smoke), while persistent hepatitis B virus infection poses particular risk for hepatoma in persons with heavy exposure to aflatoxin $(37,38)$.

Environmental cofactors influence peptic ulcer disease; cigarette smoking increases risk of ulceration. Although the exact pathogenetic mechanisms remain unknown, factors enhancing acid secretion or diminishing mucosal defenses may potentiate $H$. pylori-induced inflammation.

Duration of infection. Although gastric cancer does not occur until old age, risk for this cancer appears to be largely determined by the age of 25 . Thus, 40 or more years intercede between a critical exposure and disease outcome. Chronic infection with $H$. pylori is one plausible explanation for this long latency, and, in countries with highest gastric cancer risk, $\boldsymbol{H}$. pylori commonly infects children (2). Prolonged infection is perhaps sufficient in some circumstances for the natural progression of gastritis to malignancy. If cancer is a multistage process (8), then protracted exposure to a deleterious, potentially mutagenic process magnifies risk for disease. Sustained cell proliferation in the setting of inflammation provides continuous opportunity for cumulative mutations. Persons infected later in life may not live sufficiently long enough to see this progression to its conclusion. In other cancer models, duration of exposure to a cell growth factor also has been considered critical. In breast cancer, for example, the lifetime number of menstrual cycles determines cancer risk; multiparous women and those with later menarche have fewest cycles, lowest exposure to hormonal stimuli of cell proliferation, and lowest risk for disease (39). Similarly, hepatocellular carcinoma is most likely to develop in those with longest duration of hepatitis B infection (40).

The same childhood $H$. pylori infection that increases cancer risk, however, also would decrease risk for peptic ulcer disease. With progressive inflammation, the glandular epithelium of the stomach is impaired, eliminating the acid necessary to cause ulceration. Consequently, in countries with childhood infection, the prevalence of advanced preneoplastic lesions in young adulthood is high and the risk of ulceration is low (41). From a teleologic perspective, childhood $H$. pylori could be considered advantageous for some populations. When life expectancy is only until age 40 or 50 , childhood $H$. pylori infection, by protecting against life-threatening ulceration, would serve both the organism and the host by prolonging host survival. The brunt of $H$. pylori-related morbidity and mortality can be expected in countries undergoing industrial development with concomitant prolongation of the life span. In the United States, the peak of peptic ulcer mortality occurred in populations born during the height of the industrial revolution (42). As treatment of ulcer disease with antisecretory agents has become available, however, even this expected consequence of infection in industrialized nations has become less pronounced.

\section{Conclusions}

The role of $\boldsymbol{H}$. pylori in the pathogenesis of peptic ulceration may be a model for the effects of chronic inflammation on endocrine homeostasis (e.g., "autoimmune" thyroid diseases),
$H$. pylori-induced adenocarcinoma of the distal stomach may be relevant to other neoplasias of epithelial cells, and $H$. pylori antigen-driven lymphoid neoplasia may aid our understanding of other lymphatic malignancies. Other chronic inflammatory diseases, including Crohn's disease, multiple sclerosis, ulcerative colitis, and Wegener's granulomatosis show waxing and waning courses, often with spontaneous remissions. It is possible that any (or each) of these diseases may be caused by its own particular persistent microbial agent analogous to $H$. pylori. Instead of the all-or-none immune response leading to elimination of the microbe or to early host death that we usually consider in the pathogenesis of infectious diseases, there may be a protracted struggle in which downregulation of inflammation by both host and microbe is optimal for each, leading to a longterm homeostasis, although with ultimate clinical consequences for some. The persisting microbe may be at a distant site, but the host responses may result in recognition of cross-reacting antigens present in the affected tissue. Since regulation is crucial to this model, it may be that exogenous influences (environmental cofactors and other illnesses) reset the thermostat, leading to changes in clinical status. In other diseases in which the active component of inflammation often burns out (e.g., rheumatoid arthritis ), it is tempting to speculate that immune-mediated injury results in the loss of the natural niche for the microbe.

Persistent $H$. pylori infection may be a paradigm for other "slow" microbial processes in humans.

\section{Acknowledgments}

Work reported in this manuscript was supported by grant RO1CA 58834 from the National Cancer Institute, the Medical Research Service of the Department of Veterans Affairs, and the Society for Epidemiologic Research Merck Award.

\section{References}

1. Gotschlich, E. C. 1983. Thoughts on the evolution of strategies used by bacteria for evasion of host defenses. Rev. Infect. Dis. 5:S778-S783.

2. Taylor, D. N., and M. J. Blaser. 1991. The epidemiology of Helicobacter pylori infections. Epidemiol. Rev. 13:42-59.

3. Blaser, M. J. 1992. Hypotheses on the pathogenesis and natural history of Helicobacter pylori-induced inflammation. Gastroenterology. 102:720-727.

4. Marshall, B. J., and J. R. Warren. 1984. Unidentified curved bacilli in the stomach of patients with gastritis and peptic ulceration. Lancet. i:1311-1315.

5. Ramsey, E. J., W. L. Peterson, K. V. Carey, J. J. Jackson, F. K. Murphy, N. W. Read, K. B. Taylor, J. S. Trier, and J. S. Fordtran. 1979. Epidemic gastritis with hypochlorhydria. Gastroenterology. 76:1449-1457.

6. Thomas, J. E., G. R. Gibson, M. K. Darboe, A. Dale, and L. T. Weaver. 1992. Isolation of Helicobacter pylori from human feces. Lancet. 340:1194-1195.

7. Ihamaki, T., M. Kekki, P. Sipponen, and M. Siurala. 1985. The sequelae and course of chronic gastritis during a 30- to 34-bioptic follow-up study. Scand. J. Gastroenterol. 20:485-491.

8. Correa, P. 1992. Human gastric carcinogenesis: a multistep and multifactorial process-First American Cancer Society Award lecture on cancer epidemiology and prevention. Cancer Res. 52:6735-6740.

9. Karnes, W. E. J., I. M. Samloff, M. Siurala, M. Kekki, P. Sipponen, S. W. Kim, and J. H. Walsh. 1991. Positive serum antibody and negative tissue staining for Helicobacter pylori in subjects with atrophic body gastritis. Gastroenterology. 101:167-174.

10. Pérez-Pérez, G. I., B. M. Dworkin, J. E. Chodos, and M. J. Blaser. 1988. Campylobacter pylori antibodies in humans. Ann. Intern. Med. 109:11-17.

11. Karttunen, R., G. Andersson, K. Poikonen, T. U. Kosunen, T. Karttunen, K. Juutinen, and S. Niemela. 1990. Helicobacter pylori induces lymphocyte activation in peripheral blood cultures. Clin. Exp. Immunol. 82:485-488.

12. Blaser, M. J. 1993. Helicobacter pylori: microbiology of a "slow" bacterial infection. Trends in Microbiology. 1:255-260.

13. Dunn, B. E., G. P. Campbell, G. I. Pérez-Pérez, and M. J. Blaser. 1990 Purification and characterization of Helicobacter pylori urease. J. Biol. Chem. 265:9464-9469.

14. Pérez-Pérez, G. I., A. Z. Olivares, T. L. Cover, and M. J. Blaser. 1992. 
Characteristics of Helicobacter pylori variants selected for urease deficiency. Infect. Immun. 60:3658-3663.

15. Smith, J. T., R. E. Pounder, C. U. Nwokolo, S. Lanzon Miller, D. G. Evans, D. Y. Graham, and D. J. J. Evans. 1990. Inappropriate hypergastrinaemia in asymptomatic healthy subjects infected with Helicobacter pylori. Gut. 31:522525.

16. Mai, U. E. H., G. I. Pérez-Pérez, J. B. Allen, S. M. Wahl, M. J. Blaser, and P. D. Smith. 1992. Surface proteins from Helicobacter pylori exhibit chemotactic activity for human leukocytes and are present in gastric mucosa. J. Exp. Med. 175:517-525.

17. Mai, U. E. H., G. I. Pérez-Pérez, L. M. Wahl, S. M. Wahl, M. J. Blaser, and P. D. Smith. 1991. Soluble surface proteins from Helicobacter pylori activate monocytes/macrophages by lipopolysaccharide-independent mechanism. J. Clin. Invest. 87:894-900.

18. Peek, R. M., Jr., M. J. Blaser, and G. G. Miller. 1994. cagA-positive Helicobacter pylori strains induce preferential cytokine expression in gastric mucosa. Gastroenterology. 106:A158.

19. Blaser, M. J. 1990. Helicobacter pylori and the pathogenesis of gastroduodenal inflammation. J. Infect. Dis. 161:626-633.

20. Capron, A., and J.-P. Dessaint. 1992. Immunologic aspects of schistosomiasis. Annu. Rev. Med. 43:209-218.

21. Sharma, S. A., G. G. Miller, G. I. Pérez-Pérez, and R. S. Gupta. 1994 Humoral and cellular immune recognition of Helicobacter pylori proteins are not concordant. Clin. Exp. Immunol. In press.

22. Parsonnet, J., S. Hansen, L. Rodriguez, A. B. Gelb, R. A. Warnke, E. Jellum, N. Orentreich, J. H. Vogelman, and G. D. Friedman. 1994. Helicobacter pylori and primary gastric lymphoma. N. Engl. J. Med. 330:1267-1271.

23. Tummuru, M. K. R., T. L. Cover, and M. J. Blaser. 1993. Cloning and expression of a high molecular weight major antigen of Helicobacter pylori: evidence of linkage to cytotoxin production. Infect. Immun. 61:1799-1809.

24. Cover, T. L., C. P. Dooley, and M. J. Blaser. 1990. Characterization and human serologic response to proteins in Helicobacter pylori broth culture supernatants with vacuolizing cytotoxin activity. Infect. Immun. 58:603-610.

25. Crabtree, J. E., J. D. Taylor, J. I. Wyatt, R. V. Heatley, T. M. Shallcross, D. S. Tompkins, and B. J. Rathbone. 1991. Mucosal IgA recognition of Helicobacter pylori $120 \mathrm{kDa}$ protein, peptic ulceration, and gastric pathology. Lancet. 338:332-335.

26. Cover, T. L., and M. J. Blaser. 1992. Purification and characterization of the vacuolating toxin from Helicobacter pylori. J. Biol. Chem. 267:10570-10575.

27. Cover, T. L., M. K. R. Tummuru, P. Cao, S. A. Thompson, and M. J. Blaser. 1994. Divergence of genetic sequences for the vacuolating cytotoxin among Helicobacter pylori strains. J. Biol. Chem. 269:10566-10573.

28. Rautelin, H., B. Blomberg, H. Fredlund, G. Jarnerot, and D. Danielsson.
1993. Incidence of Helicobacter pylori strains activating neutrophils in patients with peptic ulcer disease. Gut. 34:599-604.

29. Malaty, H. M., L. Engstrand, N. L. Pedersen, and D. Y. Graham. 1993. Genetics and environment both influence Helicobacter pylori infection: the twin study. Acta Gastro-Enterol. Belg. 56:S47.

30. Boren, T., P. Falk, K. A. Roth, G. Larson, and S. Normark. 1993. Attachment of Helicobacter pylori to human gastric epithelium mediated by blood group antigens. Science (Wash. DC). 262:1892-1895.

31. Forman, D. 1991. The etiology of gastric cancer. In Relevance for Human Cancer of $\mathrm{N}$-Nitroso Compounds, Tobacco, Smoke and Mycotoxins. J. K. O'Neill, J. Chen, and H. Bartsch, editors. International Agency for Research on Cancer, Lyon, France. 22-32.

32. Howson, C., T. Hiyama, and E. Wynder. 1986. The decline in gastric cancer: epidemiology of an unplanned triumph. Epidemiol. Rev. 8:1-27.

33. Tsujii, M., S. Kawano, S. Tsuji, T. Ito, K. Nagano, Y. Sasaki, N. Hayashi, A. Fusamoto, and T. Kamada. 1993. Cell kinetics of mucosal atrophy in rat stomach induced by long-term administration of ammonia. Gastroenterology. 104:796-801.

34. Johnson, L. R., and P. P. Guthrie. 1976. Stimulation of DNA synthesis by big and little gastrin (G-34 and G-17). Gastroenterology. 1976:599-602.

35. Jackson, J. H., E. Gajewski, I. U. Schraufstatter, P. A. Hyslop, A. F. Fuciarelli, C. G. Cochrane, and M. Dizdaroglu. 1989. Damage to the bases in DNA induced by stimulated human neutrophils. J. Clin. Invest. 84:1644-1649.

36. Ames, B. N., and L. S. Gold. 1990 . Too many rodent carcinogens: mitogenesis increased mutagenesis. Science (Wash. DC). 249:970-971.

37. Gissman, L. 1984. Papillomaviruses and their association with cancer in animals and in man. Cancer Surv. 3:161-181.

38. Feitelson, M. 1992. Hepatitis B virus infection and primary hepatocellular carcinoma. Clin. Microbiol. Rev. 5:275-301.

39. Pike, M. C., D. V. Spicer, L. Dahmoush, and M. F. Press. 1993. Estrogens, progestogens, normal breast cell proliferation, and breast cancer risk. Epidemiol. Rev. 15:17-35.

40. Chisari, F. V., K. Klopchin, T. Moriyama, C. Pasquinelli, H. A. Dunsford, S. Sell, C. A. Pinkert, R. L. Brinster, and R. D. Palmiter. 1989. Molecular pathogenesis of hepatocellular carcinoma in hepatitis B virus transgenic mice. Cell. 59:1145-1156.

41. Burstein, M., E. Monge, R. Leon Barua, R. Lozano, R. Berendson, R. H. Gilman, H. Legua, and C. Rodriguez. 1991. Low peptic ulcer and high gastric cancer prevalence in a developing country with a high prevalence of infection by Helicobacter pylori. J. Clin. Gastroenterol. 13:154-156.

42. Kurata, J. H., J. D. Elashoff, B. M. Haile, and G. D. Honda. 1983. A reappraisal of time trends in ulcer disease and factors related to changes in ulcer hospitalization and mortality rates. Am. J. Public Health. 73:1066-1072. 